\title{
\begin{tabular}{l|l} 
Mibraries & DSpace@MIT
\end{tabular}
}

\author{
MIT Open Access Articles
}

The double-walled nature of $\mathrm{TiO}_{2}$ nanotubes
and formation of tube-in-tube structures - a
characterization of different tube morphologies

The MIT Faculty has made this article openly available. Please share how this access benefits you. Your story matters.

Citation: So, Seulgi et al. "The double-walled nature of $\mathrm{TiO}_{2}$ nanotubes and formation of tube-intube structures - a characterization of different tube morphologies." Electrochimica Acta, 231 (February 2017): 721-731 @ 2017 The Author(s)

As Published: 10.1016/J.ELECTACTA.2017.02.094

Publisher: Elsevier BV

Persistent URL: https://hdl.handle.net/1721.1/127256

Version: Author's final manuscript: final author's manuscript post peer review, without publisher's formatting or copy editing

Terms of use: Creative Commons Attribution-NonCommercial-NoDerivs License 


\section{The double-walled nature of $\mathrm{TiO}_{2}$ nanotubes and formation of tube-in-tube structures - a characterization of different tube morphologies}

Seulgi So, ${ }^{1,2 \#}$ Francesca Riboni, ${ }^{1 \#}$ Dennis Paul, ${ }^{3}$ John Hammond, ${ }^{3}$ Ondrej Tomanec,${ }^{4}$ Radek Zboril, ${ }^{4}$ Donald R. Sadoway, ${ }^{2}$ Patrik Schmuki ${ }^{1,5 *}$

\footnotetext{
${ }^{1}$ Department of Materials Science WW4-LKO, University of Erlangen-Nuremberg, Martensstrasse 7, 91058 Erlangen, Germany

${ }^{2}$ Department of Materials Science and Engineering, Massachusetts Institute of Technology, 77

Massachusetts Avenue, Cambridge, Massachusetts 02139-4307, USA

${ }^{3}$ Physical Electronics USA, 18725 Lake Drive East, Chanhassen, MN, US

${ }^{4}$ Regional Centre of Advanced Technologies and Materials, Šlechtitelů 27, 78371 Olomouc, Czech

Republic

${ }^{5}$ Department of Chemistry, Faculty of Science, King Abdulaziz University, P.O. Box 80203, Jeddah

21569, Saudi Arabia

\# These authors contributed equally to this work

*Corresponding author. E-mail: schmuki@ww.uni-erlangen.de
}

Link to the published article:

https://www.sciencedirect.com/science/article/pii/S0013468617303705 


\begin{abstract}
In the present work we show how to achieve different nanotube morphologies (namely, not only single- and double-walled tubes, but also a tube-in-tube configuration) by combining specific anodization parameters. We characterize as-grown tube layers in terms of morphology, chemical composition and properties. As-grown tubes exhibit a double-walled morphology, that is, they consist of an inner and an outer shell. The low quality inner shell can be removed by an optimized chemical treatment thus leading to nanotubes of a higher quality oxide and with a single-walled nature. Double-walled tubes grown at low electrolyte temperature provide a thick inner shell that after adequate annealing can form a unique tube-in-tube morphology.
\end{abstract}

\title{
Keywords
}

Double-walled nanotubes, single-walled nanotubes, tube-in-tube configuration, ThermoGravimetric Analysis, Differential Scanning Calorimetry 


\section{Introduction}

One-dimensional (1D) nanostructures, in particular in the form of nanotubes (NTs), provide an exceptional combination of optical, electrical and chemical properties with a distinct and defined geometry. Therefore, they find application in fields such as energy conversion and storage, and medicine. ${ }^{1-3}$

Several methods are reported for the synthesis of $1 \mathrm{D} \mathrm{TiO}_{2}$ nanotubes, such as hydrothermal, solgel or template-assisted approaches. ${ }^{4-6}$ The most common and straightforward is self-organizing electrochemical anodization $(\mathrm{SOA}){ }^{7,3,8}$

Traditionally, anodization is carried out in a simple two-electrode configuration, where the metal of interest $\left(\mathrm{M}\right.$, the anode) under a suitable voltage is oxidized $\left(\mathrm{M} \rightarrow \mathrm{M}^{z+}+z \mathrm{e}^{-}\right)$and converted to the corresponding (mostly compact) metal oxide, by a reaction with $\mathrm{O}^{2-}$ ions derived from $\mathrm{H}_{2} \mathrm{O}$ in the electrolyte. ${ }^{3}$

For an optimized set of experimental parameters (e.g., electrolyte composition and $\mathrm{pH}$, temperature, applied voltage, time etc.), self-organizing conditions can be established and onedimensional $\mathrm{TiO}_{2}$ NTs can be grown as highly aligned, size-controlled, and back-contacted (i.e., anchored to a metallic substrate) nanostructure arrays. These arrays provide a broad range of functionalities and properties $e . g$. for use in solar cells,,${ }^{9,10}$ as well as in photo-electrochemical, ${ }^{11}$ energy storage ${ }^{12}$ and biochemical devices. ${ }^{13}$ Key to the formation of nanoporous/nanotubular oxide arrays is the presence of $\mathrm{F}^{-}$ions in the electrolyte that, under optimized conditions, establishes a steady-state equilibrium between oxide formation and dissolution required for selforganization. ${ }^{14,15}$

Since the first demonstration of self-ordered anodic $\mathrm{TiO}_{2}$ nanostructures by anodization, ${ }^{16}$ tremendous research on the structural design and properties of anodic $\mathrm{TiO}_{2}$ tubes has been carried out. 
The first generation of anodic $\mathrm{TiO}_{2}$ NTs was grown in acidic-aqueous solutions. ${ }^{16-18}$ For these tubes, only a limited maximum length $(\sim 2.5 \mu \mathrm{m}$, due to high chemical etching rate promoted by excess water) and a low self-ordering degree (ripple-walled tubes were typically reported) could be achieved.

The introduction of organic electrolytes represents a keystone in the growth of smooth, ordered and longer (up to several $100 \mu \mathrm{m}$ ) $\mathrm{TiO}_{2}$ nanotubes. ${ }^{19}$ Among organic electrolytes, most classic are glycerol and the nowadays widely used mixtures of ethylene glycol (EG) containing ammonium fluoride $\left(\mathrm{NH}_{4} \mathrm{~F}\right)$ and low amounts of water ( 1-5 vol\%). Tubes grown in these electrolytes typically feature a double-walled (DW) structure, with an inner wall rich in carbon-species embedded from the electrolyte during the anodic growth. ${ }^{20-22}$

Owing to its composition, ${ }^{22}$ the inner shell is notoriously detrimental for $\mathrm{TiO}_{2} \mathrm{NT}$ applications e.g. as photo-anode in solar cells and photo-electrochemical devices, among others, as carbon retards the electron mobility.

Most recent findings show that a simple chemical treatment in combination with lowtemperature annealing can selectively etch out the inner part of DW NTs leaving behind a so-called single-walled (SW) nanostructure; SW tubes typically have larger inner diameter, smoother and better-defined wall surface and, most notably, improved electronic properties compared to the original DW tubes. ${ }^{22}$

A most remarkable step-forward in the fabrication of $\mathrm{TiO}_{2}$ nanotubes has been achieved through the introduction in the traditional EG-based electrolyte of weak organic acids (e.g., lactic acid (LA), glycolic acid, citric acid, and EDTA). By preventing the occurrence of dielectric breakdown events in the growing oxide, these additives allow anodization at a significantly higher voltage, ultimately yielding a ultra-fast growth that drastically (positively) affects the tubes' properties. Specifically, while preserving a double-walled morphology, EG/LA- $\mathrm{TiO}_{2}$ NTs exhibit a larger diameter, 
superior mechanical resistance, and improved robustness, compared to classic EG-tubes. All these features contribute to an amelioration of the performance of such nanotubes in several applications. $^{22-25}$

In spite of the wide use of DW- $\mathrm{TiO}_{2} \mathrm{NTs}$ fabricated in EG/LA-based electrolytes, their structural and chemical properties as well as their differences to SW tubes have only sparingly been investigated.

Thus, within this work, we characterize composition, structure and differences in properties of such tubes.

Moreover, we find that, for tubes grown in EG/LA electrolyte using low anodizing temperatures, a significant thickening of the NT walls is observed; this then represents a precursor state to form a remarkable tube-in-tube (T-in-T) morphology that develops during an annealing treatment.

\section{Experimental}

\subsection{Preparation of $\mathrm{TiO}_{2}$ nanotubes}

$\mathrm{TiO}_{2}$ nanotubes $(16 \mu \mathrm{m}$-long) were grown on titanium foils $(0.125 \mathrm{~mm}$ thick, $99.6+\%$ purity, Advent, England) that, prior to anodization, were degreased by sonication in acetone, ethanol and isopropanol, rinsed with deionized water, and then dried with a nitrogen jet. Electrochemical anodization was performed with a high-voltage potentiostat (Jaissle IMP 88 PC) at 120 V in a twoelectrode configuration with a Pt foil as a counter electrode, and using an electrolyte composition of 1.5 M lactic acid (LA, DL-Lactic acid, 90\%, Fluka), $0.1 \mathrm{M}$ ammonium fluoride $\left(\mathrm{NH}_{4} \mathrm{~F}\right)$ and 5 wt $\%$ deionized $\mathrm{H}_{2} \mathrm{O}$ in ethylene glycol $(99 \mathrm{vol} \%){ }^{23}$

Anodization was carried out at two different temperatures, namely room temperature (RT) and $10^{\circ} \mathrm{C}$ (set with a HAAKE F3 Thermostat). In particular, $16 \mu \mathrm{m}$-long tubes were grown in $10 \mathrm{~min}$ 
for anodizing at RT, while much longer time (1h $30 \mathrm{~min}$ ) was needed to obtain comparably long tubes at $10^{\circ} \mathrm{C}$.

After anodizing, the samples were washed in ethanol and dried in a nitrogen stream.

Thereafter, a chemical dissolution procedure was used to selectively dissolve the low-quality tube inner shell. ${ }^{22}$

For this, RT-nanotubes were first annealed in a Rapid Thermal Annealer (Jipelec JetFirst100) at $150^{\circ} \mathrm{C}$ for $1 \mathrm{~h}$ in air, at a heating and cooling rate of $30^{\circ} \mathrm{C} \mathrm{min}{ }^{-1}$. Afterwards, tubes were dipped in a piranha solution $\left(\mathrm{H}_{2} \mathrm{SO}_{4}: \mathrm{H}_{2} \mathrm{O}_{2}=3: 1\right)$ for 6 min at $70^{\circ} \mathrm{C}$, then washed in $\mathrm{H}_{2} \mathrm{O}$ and EtOH, and finally dried with a nitrogen jet.

Some of the fabricated tube layers (prepared either at RT (both single- and double-walled tubes) or at $10^{\circ} \mathrm{C}$ ) were annealed in a Rapid Thermal Annealer at $450{ }^{\circ} \mathrm{C}$ in air for $1 \mathrm{~h}$, with a heating and cooling rate of $30^{\circ} \mathrm{C} \mathrm{min}^{-1}$.

\subsection{Material physico-chemical characterization}

For morphological characterization, a field-emission scanning electron microscope (FESEM, Hitachi SEM FE 4800) was used. The thickness of the nanotubes was measured from SEM crosssection images.

X-ray diffraction (XRD), performed with an X'pert Philips MPD (equipped with a Panalytical $\mathrm{X}^{\prime}$ celerator detector) using graphite monochromized $\mathrm{Cu} \mathrm{K} \alpha$ radiation $(\lambda=1.54056 \AA$ ), was used to analyze the crystallographic properties of the materials.

HRTEM images were obtained using a HRTEM TITAN 60-300 microscope with an X-FEG type emission gun, operating at $300 \mathrm{kV}$. This instrument is equipped with a Cs image corrector and a STEM/HAADF detector; its point resolution is $0.06 \mathrm{~nm}$ in the TEM mode. The elemental mappings were obtained by STEM/EDS technique, with an acquisition time of $20 \mathrm{~min}$. 
Composition and chemical state information were obtained by Energy Dispersive X-ray (EDX) and by Scanning Auger Nanoprobe (PHI 710 - Physical electronics, US; accelerating voltage 20 $\mathrm{kV}$, current $1 \mathrm{nA})$.

High spatial resolution scanning Auger Electron Spectroscopy (AES, Physical Electronics PHI 700Xi Scanning Auger Nanoprobe) was employed to investigate the compositional variations along the $\mathrm{TiO}_{2}$ nanotube length.

Thermogravimetric analysis (TGA) coupled to Mass Spectrometry (MS) (Netzsch STA 409 CD, equipped with a Skimmer QMS 422 mass spectrometer (MS/EI)) was carried out to study the thermodynamic behavior of the samples. $\mathrm{TiO}_{2}$ nanotubes (around $10 \mathrm{mg}$ ) were detached from $\mathrm{Ti}$ metal foil by physical bending of the substrate. TGA-MS was scanned from room temperature (RT) to $650^{\circ} \mathrm{C}$, with a $10^{\circ} \mathrm{C} / \mathrm{min}$ ramping rate, in air. To remove contaminations, helium was purged before any measurement.

Fourier Transform Infrared spectroscopy (FTIR; Thermo Fisher FTIR6700) was carried out with a microscope attached to a FTIR6700 bench. FTIR spectra were collected from a $c a .100 \mu \mathrm{m}-$ large spot.

Differential Scanning Calorimetry (DSC, Perkin Elmer) was used to measure the crystallization temperature of the investigated samples. $\mathrm{TiO}_{2}$ nanotubes $(\sim 10 \mathrm{mg})$ were first detached from the $\mathrm{Ti}$ metal foil by physical bending of the substrate; spectra were collected in a $\mathrm{N}_{2}$ atmosphere and the temperature was scanned from $25^{\circ} \mathrm{C}$ to $400^{\circ} \mathrm{C}$, with a $10^{\circ} \mathrm{C} / \mathrm{min}$ ramping rate.

\section{Results and Discussion}

Nanotube layers, as shown in Fig. 1, were fabricated in an organic-based electrolyte (0.1 M $\mathrm{NH}_{4} \mathrm{~F}-5$ wt. $\left.\% \mathrm{H}_{2} \mathrm{O}-\mathrm{EG}\right)$, also containing lactic acid (1.5 M). 
This electrolyte was selected as lactic acid is reported to shift the "burning" voltage to a significantly higher value ${ }^{26}$ and, thus, allows a high ion flux through the oxide film and therefore provides an extremely fast growth of the anodic oxide without affecting the tubes' functional properties. ${ }^{23,27,28}$

Anodizing in EG/LA electrolyte at room temperature leads to $16 \mu \mathrm{m}$-thick nanotube layers in $10 \mathrm{~min}$ - remarkably, anodization time longer than $2 \mathrm{~h}$ is typically required to grow comparably long $\mathrm{TiO}_{2} \mathrm{NTs}$ in traditional EG-based electrolytes with no lactic acid. ${ }^{23}$

As shown in Fig.1 (a-c), $\mathrm{TiO}_{2}$ nanotubes grown under these conditions are double-walled nanotubes that consist of two different types of oxide, namely an outer shell (OS) of comparably pure $\mathrm{TiO}_{2}$, and an inner shell (IS) that is mainly composed of carbon-rich titanium oxide or oxyhydroxide and that forms due to electrolyte uptake during anodization. ${ }^{20-22}$

Despite a clear difference in quality and composition, the outer and inner shells of DW nanotubes cannot be clearly distinguished from SEM in as-grown layers (Fig. 1 (a)).

However, annealing of the DW NTs leads to a separation of IS and OS into two distinct layers; in detail, the outer layer remains compact, while the inner shell becomes porous (Fig. $1(b, c))$.

This is an indication that carbon-trapped species (hydrocarbon compounds) are mainly in the IS, and being "burned-off" and evaporated as $\mathrm{CO}_{2}$ during annealing produces the observed porosity - in line with literature ${ }^{29}$.

De-coring of the DW tubes of Fig. 1(a) as described in the experimental section leads to singlewalled nanotubes (Fig. 1 (d)) that exhibit both a thinner wall and a wider inner diameter $(\sim 20 \mathrm{~nm}$ and $~ 95 \mathrm{~nm}$, respectively).

These results were obtained after optimizing the experimental conditions of the de-coring (etching) process, namely the immersion temperature and time to $70^{\circ} \mathrm{C}$ and $6 \mathrm{~min}$, respectively. 
For longer etching (and/or higher temperatures) the resulting dissolution of the OS significantly affects the morphology and, eventually, the $\mathrm{TiO}_{2}$ nanotubes peel off from the Ti substrate.

Annealing of single-walled tubes (i.e., consisting of only the OS) does, in contrast to the DW tubes, not affect the morphology of the layer as observed in SEM (Fig. 1(e,f)) and reflects the high quality (and thermal resistance) of the oxide.

As shown by XRD patterns in Fig. 2a, as-formed tubes are amorphous and annealing under most classic conditions for EG-based tubes $\left(450^{\circ} \mathrm{C}, 1 \mathrm{~h}\right.$ in air) leads to the crystallization of both DW and SW layers in the form of nearly pure anatase, with only a small contribution from rutile (Fig. 2(b)).

Information on the chemical compositions of double- and single-walled tubes has been obtained from a range of characterization techniques, as described below.

Energy Dispersive X-ray measurements indicate that as-formed DW tubes exhibit a content of carbon higher than that of single-walled NTs (i.e., $~ 7.5$ at $\%$ and $\sim 5.5$ at. $\%$ respectively, Fig. 3(a)), this being attributed to the presence of the inner tube shell in the double-walled morphology; similar amounts of $\mathrm{F}$ were observed in both $\mathrm{DW}$ and $\mathrm{SW}$ - that is, $\mathrm{F}^{-}$is mainly in the outer layer and originates from anodizing in the presence of fluorides (here, $\mathrm{NH}_{4} \mathrm{~F}$ ).

More detailed investigations were carried out on ion-milled cross-sections of amorphous tubes. Fig. 3(b,c) shows the line scans of titanium, fluoride and carbon collected through Scanning Auger Electron spectroscopy and superimposed on the original SEM images of the different tube morphologies.

In line with a double-layered morphology, $\mathrm{C}$ distribution in DW structures is higher in the inner walls of the tubes. On the other hand, Ti and F concentrations drop inside the tubes, with F-rich regions corresponding to $\mathrm{C}$-depleted regions (and vice versa). ${ }^{23,30}$ 
In particular, AES of DW structures points out that the concentration of $\mathrm{F}$ is highest in the outermost part of the outer layer, in line with a comparably higher concentration of $\mathrm{F}^{-}$in-between tubes. This is in line with earlier findings and anodic tube formation mechanisms that lead to a fluoride-rich layer at tube bottoms and outer walls during nanotube growth. ${ }^{30,31}$

In SW tubes, Ti and C concentrations reach their local maxima in the walls (OS) and decrease inside the tubes (Fig. 3(c)); this confirms the removal of the carbon-rich shell by chemical etching in piranha solution..$^{20,21,29}$

High-angle annular dark field scanning TEM (HAADF-STEM) and elemental mapping of amorphous DW tubes (Fig. 4(a)) show a distribution of C, F and TiO in line with that revealed by AES: carbon-species (from electrolyte uptake) mainly concentrate in the inner tube shell, while the amounts of fluoride and titanium (oxide or oxy-hydroxide) are higher towards the tubes' outer walls.

HAADF and TEM mappings of SW tubes in Fig. 4(b) confirm the removal of the inner shell after chemical etching, with a consequent significant decrease of the wall overall thickness. Moreover, $\mathrm{C}$ (intrinsic residues), $\mathrm{F}$ and $\mathrm{TiO}$ species are homogeneously distributed in the remaining outer shell.

For anodization performed at $10^{\circ} \mathrm{C}$, a significantly longer time (1h $30 \mathrm{~min}$ vs. $10 \mathrm{~min}$ at $\mathrm{RT}$, Fig. 2(c)) was needed to grow $16 \mu$ m-long nanotubes (Fig. 1 (g)); that is, the nanotubes' growth rate decreases by a factor of 9 .

This is mainly ascribed to the lower anodizing temperature that influences the kinetics of ion migration through the oxide, as well as the solubility of the reaction products in the electrolyte. A combination of these factors causes a two-fold effect: i) $\mathrm{Ti}^{4+}, \mathrm{O}^{2-}$ and $\mathrm{F}^{-}$migration (key to a steadystate oxide formation/dissolution equilibrium for self-organization) is slowed down - hence, longer 
anodization time is clearly needed, but also ii) the solubility of Ti hydroxides, oxy-hydroxides and C-enriched compounds (embedded from the electrolyte) is reduced. ${ }^{32,33}$

Therefore, under such conditions, an overall significant thickening of IS is observed, as shown in Fig. 1(g), and Ti-C based compounds (formed by reaction of carbon residues and precipitated Ti ions ejected from the growing oxide) accumulate in the IS.

From Fig. 1(g), as-formed tubes grown at $10^{\circ} \mathrm{C}$ show $\sim 80 \mathrm{~nm}$-thick walls and an inner diameter of $\sim 30 \mathrm{~nm}$, while those prepared at RT have $\sim 45 \mathrm{~nm}$-thick walls and a $\sim 55 \mathrm{~nm}$ inner diameter.

For these tubes, after annealing at $450^{\circ} \mathrm{C}$ in air, a remarkable tube-in-tube morphology as shown in Fig. 1(h,i) becomes apparent. The tube wall thickness is reduced (i.e., from $\sim 80 \mathrm{~nm}$ to $\sim 48 \mathrm{~nm}$ ) and an additional inner tubular structure is formed.

Elemental composition of the as-formed precursor tubes (i.e., T-in-T(prec) that after annealing become tube-in-tube structure) by EDX (Fig. 3(a)) shows that T-in-T(prec) exhibit the highest content of carbon and also of fluoride species (i.e., 10 at $\%$ and $~ 7$ at $\%$, respectively,), in line with the proposed mechanism based on increased ion accumulation in the IS at low anodization T.

Concentration scans of fluoride, titanium and carbon obtained by scanning AES and superimposed on the ion-milled SEM image of T-in-T(prec) in Fig. 3(d) are also in line with the above scenario. That is, the distributions of $\mathrm{Ti}$ and $\mathrm{F}$ appear nearly uniform throughout the nanotubes, while that of $\mathrm{C}$ is higher inside the tubes, thus confirming the accumulation of a carbonrich Ti-based layer in the tubes' inner shell, ${ }^{32,33}$ due to the low solubility of the IS at $10^{\circ} \mathrm{C}$.

TEM mappings of T-in-T(prec) (Fig. 4(c)) confirm a nearly homogeneous distribution of C, F and $\mathrm{TiO}$ in the tube walls, and a comparison of the HAADF-STEM images of all the as-formed samples (Fig. 4(a-c)) clearly highlights that overall the thickness of NT walls follows the order Tin-T(prec) $>$ DW $>$ SW . 
Moreover, a comparison of the fluoride distribution and content in T-in-T before and after annealing (i.e., T-in-T(prec) vs. T-in-T(ann)) at $450^{\circ} \mathrm{C}(\mathrm{Fig} .5(\mathrm{a}, \mathrm{b}))$ shows a lower $\mathrm{F}$ content in the annealed sample suggesting that the fluoride accumulated inside the tubes (Fig. 3(d)) is partially removed during the thermal treatment. This may be attributed to the sublimation of $\operatorname{TiF}_{x}$ compounds that typically occurs at $\mathrm{T} \sim 290^{\circ} \mathrm{C} .^{34}$

In line with this, EDX of crystalline T-in-T (Fig. 3(a), T-in-T(ann)) shows that F and also C contents drastically drop to $\sim 2-4$ at.\% (that is, the level of environmental contamination), ${ }^{22}$ and the TEM image in Fig. 5(c) clearly shows the appearance of an inner tube and of empty space after annealing the T-in-T(prec).

The compositional and structural changes associated with the annealing process in DW, SW, and T-in-T(prec) were followed using Thermo-Gravimetric Analysis, coupled with Mass Spectrometry (TGA-MS); the characteristic T-dependent decomposition patterns are shown in Fig. 6.

A 2-3 wt\% loss (corresponding to a mass/charge $(\mathrm{m} / \mathrm{e})=18)$ is observed in all the three layers at $\mathrm{T} \sim 250-300^{\circ} \mathrm{C}$, that may be ascribed to $\mathrm{H}_{2} \mathrm{O}$ desorption from uncoordinated Ti surface atoms. ${ }^{35}$

Different responses for the three tubular layers are observed for a mass/charge loss of 44 (i.e., corresponding to $\mathrm{CO}_{2}$ ), in the range of $\mathrm{T} \sim 380-390^{\circ} \mathrm{C}$. It is reported that desorption of environmental $\mathrm{CO}_{2}$ from $\mathrm{TiO}_{2}$ surface typically occurs at room temperature (or below). ${ }^{36,37}$ Therefore, it is plausible that the carbon loss observed by TGA at the higher temperatures corresponds to the pyrolysis of some C-based contaminants in all the three NT layers.

In particular, the lowest weight loss $(\sim 5 \mathrm{wt} \%$, Fig. $6(\mathrm{~b}))$ is observed for single-walled tubes; in this case, carbon loss is assigned to the combustion of some contaminants from the tube outer shell that remain also after de-coring DW tubes and that can be removed from SW tubes only when annealing at $\mathrm{T} \sim 400^{\circ} \mathrm{C}$ or higher. ${ }^{20,22}$ 
Higher amounts of carbon (i.e., 8-9 wt\%, Fig. 5(a,c)), in line with data from EDX (Fig. 3(a)), are "burned-off" in double-walled and tube-in-tube NTs. Here, the contribution is dominated by the thermal oxidation of carbon species embedded in the tube inner walls (see above). ${ }^{20}$ Moreover, the larger amount of $\mathrm{CO}_{2}$ from $\mathrm{T}$-in- $\mathrm{T}$ with respect to DW supports the proposed mechanism of wall thickening at low anodization $\mathrm{T}$ due to electrolyte uptake and accumulation/reduced ion solubility.

A most significant difference in the $\mathrm{CO}_{2}$ peak for the three tube configurations is reported in Fig. 6(d).

It is clear that the combustion of $\mathrm{C}$ species in DW and T-in- $\mathrm{T}$ tubes occurs at a lower temperature than in SW; this, together with a sharper peak profile than in the SW case, indicates a possible different nature of carbon compounds in the structures.

FTIR spectra collected for as-formed DW, SW and T-in-T, and reported in Fig. 7(a) confirm the presence of $\mathrm{OH}-$ and of $\mathrm{C}$-based moieties on the surface of the three types of nanotubes.

In all the three cases, the peaks at $1620-1630 \mathrm{~cm}^{-1}$ and the broad peaks at $3100-3600 \mathrm{~cm}^{-1}$ are assigned to the bending and stretching modes, respectively, of hydroxyl groups ${ }^{38}$ In particular, the broad band at $\sim 3300 \mathrm{~cm}^{-1}$ and the relatively sharper band at $\sim 1650 \mathrm{~cm}^{-1}$ can be assigned to the $\mathrm{OH}$ stretching and the $\mathrm{HOH}$ bending modes of free water, respectively, ${ }^{39}$ while the peaks in the $3400-3600 \mathrm{~cm}^{-1}$ correspond to $-\mathrm{OH}$ groups weakly bound to uncoordinated Ti surface sites. ${ }^{40}$

For DW and T-in-T structures well defined peaks appear at $2840-3000 \mathrm{~cm}^{-1}$ corresponding to alkyl $-\mathrm{CH}_{2}$ and $-\mathrm{CH}$ stretching bonds ${ }^{39}$ likely due to EG/LA residues. Accordingly, the intensity of the carbon-associated vibration modes in single-walled NTs is significantly lower compared to that of the other two structures (DW and T-in-T tubes). ${ }^{41,42}$

Finally, the vibrational modes associated to environmental $\mathrm{CO}_{2}$ adsorption $\left(\sim 2340 \mathrm{~cm}^{-1}\right)$ appear in all three as-formed layers. ${ }^{43,44}$ 
Additional FTIR spectra (Fig. 7(b)) have been collected to identify differences in as-formed (i) traditional DW tubes (DW(ref)), (ii) DW tubes with (purposely unremoved) electrolyte remnants (i.e., no washing-step in EtOH after anodization - DW(no EtOH)), and (iii) DW tubes before etching treatment (DW(Ann150)). The origin of C-contamination is of primary concern.

All the DW layers exhibit similar FTIR spectra, both in terms of band positions and relative intensities.

A most relevant aspect is the similar surface compositions of DW(ref) and DW(no EtOH) layers: this confirms that $\mathrm{C}$ content in DW structures is not the result of plain electrolyte residues in the oxide tubes - in this case, washing in EtOH would have removed this contribution at least partially, and DW(ref) and DW(noEtOH) spectra would exhibit different features.

Moreover, a $\mathrm{C}$ content (from the peak relative intensities) that follows the trend DW(Ann150) $\sim \mathrm{DW}($ ref $)>\mathrm{SW}($ Fig. $7(\mathrm{a}, \mathrm{b}))$ confirms that (i) mild annealing at $150^{\circ} \mathrm{C}$ before the chemical etching treatment does not affect the C contained in DW tubes (DW(Ann150) DW(ref)) and (ii) the main contribution to the overall carbon content comes from the inner shell (DW(ref) $>$ SW).

Information on thermally-induced transitions (namely, crystallization) in DW, SW and T-in$\mathrm{T}$ (prec) structures was obtained from Differential Scanning Calorimetry measurements (Fig. 8(a)).

Noteworthy, data from DSC are complementary to those from TGA: the thermogravimetric analysis provides information on mass variation (e.g., due to dehydration and combustion processes) occurring in a material heated up to a certain T; on the other hand, DSC shows the material response to a temperature variation in terms of lattice rearrangement. ${ }^{45}$

In particular, DSC peaks as those reported in Fig. 8(a) indicate that the material undergoes crystallization, with the $\mathrm{T}$ of maximum heat flow representing the crystallization temperature $\left(\mathrm{T}_{\mathrm{c}}\right)$. Typically, the most intense the DSC peaks, the fastest is the associated reaction - that is, the less defective the material. 
Fig. 8(a) shows that not only single-walled nanotubes exhibit the lowest crystallization temperature, but also feature the sharpest and most intense peak; in line with previous discussion, both characteristics prove the high-quality oxide composition of the outer tube shell and indicate that the amorphous-into-crystalline transition in SW tubes is already achieved at $\mathrm{T}_{\mathrm{c}}$ as low as $356^{\circ} \mathrm{C}$.

By contrast, both DW and T-in-T tubes undergo crystallization at higher temperatures (i.e., $\mathrm{T}_{\mathrm{c}}$ $=c a .363$ and $375^{\circ} \mathrm{C}$, respectively), and the broadening of the associated DSC peaks suggest that parallel processes occur in combination with crystallization.

In particular, $\mathrm{T}_{\mathrm{c}}$ values of $\mathrm{DW}$ and $\mathrm{T}$-in- $\mathrm{T}$ tubes are in the same temperature range as that of carbon (hydrocarbon) species combustion to $\mathrm{CO}_{2}$ determined by TGA (Fig. 6(d)).

It is thus plausible that the large amount of carbon accumulated in DW and T-in-T tubes retard the crystallization of the tubes.

By contrast, the $\mathrm{T}_{\mathrm{c}}$ value of $\mathrm{SW}$ tubes is clearly lower than the temperature for $\mathrm{CO}_{2}$ release from such structures (Fig. 6(b)), indicating that carbon remnants in the outer shell of tubes do not affect the crystallization process. Nevertheless, it should be mentioned that $\mathrm{F}^{-}$is known to facilitate the amorphous-to-crystalline transition of $\mathrm{TiO}_{2} .{ }^{46}$ Therefore, a contribution of fluoride from the OS (Fig. 4(b)) to the lowering of the crystallization temperature for SW structures cannot be excluded.

The different responses to $\mathrm{T}$ variation of the three NT types confirm the different origins and properties of C-based species and further highlight the close similarity between DW and T-in-T precursor structures.

DSC characterization of the additional DW(no EtOH) and DW(Ann150) nanotube layers (Fig. 8(b)) is also in line with the above results: the similar peaks obtained with the three different double-walled NT samples show that the retarded crystallization process should be solely attributed 
to the intrinsic composition of the IS in the double-walled structures, with no contribution from adsorbed electrolyte species.

Most important, results in Fig. 8(b) confirm that only chemical etching of DW tubes under optimized conditions can lead to the recovery of properties and chemical composition that are typical of higher-quality $\mathrm{TiO}_{2}$, by removing the poor-quality $\mathrm{C}$-enriched inner shell.

\section{Conclusion}

A most remarkable key-aspect, pointed out by above results, is that, although annealing of DW and T-in-T(prec) structures leads to two clearly different morphologies, namely the formation of a porous inner shell and that of self-standing inner tubes, respectively, all the characterizations carried out show similar chemical composition and properties of the two morphologies that set them apart from SW NTs.

In particular, EDX measurements and TEM elemental mappings (Fig. 3 and 4) showed that double-walled and tube-in-tube layers not only feature comparable chemical composition (i.e., both contain C (and F) species accumulated from the electrolyte), but these species have also similar distribution profiles within the tubes as, in both cases, they were shown to accumulate in the inner shell.

Moreover, TGA and DSC results indicated that owing to the peculiar composition of their double-walled structure, dehydration and combustion processes occur when heating the layers (more precisely, they occur at a larger extent than in SW tubes) and, most important, showed that the presence of significant amounts of $\mathrm{C}$ retards the crystallization process.

A most relevant (and only) difference between amorphous DW and T-in-T is, therefore, the relatively larger thickness of the T-in-T walls, owing to anodizing at low temperature - this being particularly evident from the SEM images in Fig. 1. 
Therefore, also in line with previous studies, ${ }^{32,33}$ one can conclude that the same anodic mechanism is at the basis of both DW and T-in-T(prec) growth, and may tentatively ascribe the origin of the peculiar tube-in-tube configuration to a thermally-induced effect.

Annealing of the double-walled nanotubes leads to the porosification of the inner shell (see SEM images of Fig. 1(b,c)), due to the sublimation/evaporation of $\mathrm{C}$ species confirmed by TGA analysis (Fig. 6(a)). At sufficiently high temperature $\left(\sim 400^{\circ} \mathrm{C}\right)$, IS carbon residues are burned-off as $\mathrm{CO}_{2}$, which evaporates most likely by diffusing through the $\sim 20 \mathrm{~nm}$-thick inner layer (Fig. 1(b)) and by leaving the nanotubular structure from the inner cavity and leading to a mild separation of IS and OS.

However, the relatively larger thickness of the IS of T-in-T precursor structures compared to that in DW tubes (Fig. 1(a,d)) may lead to a wider separation of the inner shell from the outer shell and to the appearance of clear self-standing inner tube (Fig. 1(h,i)).

We therefore propose that the formation of inner tubes may be promoted by low temperature anodization and thick IS formation, in combination with adequate annealing. More precisely, the low anodizing $\mathrm{T}$ establishes a reduced ion mobility and solubility that lead to the observed thickening of IS, while annealing promotes the detachment of the inner shell from the outer shell and, as a consequence, leads to the formation of (smaller) nanotubes into (larger) nanotubes. 


\section{Acknowledgements}

The authors would like to acknowledge ERC, DFG and the DFG “cluster of excellence" EAM for financial support.

\section{References}

1 C. M. Lieber, Solid State Commun., 1998, 107, 607-616.

2 C. N. R. Rao, A. Muller and A. K. Cheetham, The Chemistry of Nanomaterials: Synthesis, Properties and Applications., Weinheim, Germany, 2004.

3 K. Lee, A. Mazare and P. Schmuki, Chem. Rev., 2014, 114, 9385-9454.

4 T. Kasuga, M. Hiramatsu, A. Hoson, T. Sekino and K. Niihara, Langmuir, 1998, 14, 3160 3163.

5 M. S. Sander, M. J. Côté, W. Gu, B. M. Kile and C. P. Tripp, Adv. Mater., 2004, 16, 20522057.

6 M. S. Sander and H. Gao, J. Am. Chem. Soc., 2005, 127, 12158-12159.

$7 \quad$ P. Roy, S. Berger and P. Schmuki, Angew. Chemie - Int. Ed., 2011, 50, 2904-2939.

8 F. Riboni, N. T. Nguyen, S. So and P. Schmuki, Nanoscale Horizons, 2016, 1, 445-466.

9 K. Zhu, T. B. Vinzant, N. R. Neale and A. J. Frank, Nano Lett., 2007, 7, 3739-3746.

10 T. Stergiopoulos, A. Ghicov, V. Likodimos, D. S. Tsoukleris, J. Kunze, P. Schmuki and P. Falaras, Nanotechnology, 2008, 19, 235602.

11 C. Das, P. Roy, M. Yang, H. Jha and P. Schmuki, Nanoscale, 2011, 3, 3094-3096.

12 A. Ghicov, S. P. Albu, J. M. Macak and P. Schmuki, Small, 2008, 4, 1063-1066.

13 J. Park, S. Bauer, K. Von Der Mark and P. Schmuki, Nano Lett., 2007, 7, 1686-1691.

14 J. M. Macak, H. Tsuchiya, A. Ghicov, K. Yasuda, R. Hahn, S. Bauer and P. Schmuki, Curr. Opin. Solid State Mater. Sci., 2007, 11, 3-18.

15 S. P. Albu, A. Ghicov, J. M. Macak and P. Schmuki, Phys. status solidi-Rapid Res. Lett., 2007, 1, R65-R67.

16 M. Assefpour-Dezfuly, C. Vlachos and E. H. Andrews, J. Mater. Sci., 1984, 19, 3626-3639.

17 V. Zwilling, E. Darque-Ceretti, A. Boutry-Forveille, D. David, M. Y. Y. Perrin and M. Aucouturier, Suface Interface Anal., 1999, 27, 629-637.

18 R. Beranek, H. Hildebrand and P. Schmuki, Electrochem. Solid-State Lett., 2003, 6, B12B14.

19 J. M. Macak, H. Tsuchiya, L. Taveira, S. Aldabergerova and P. Schmuki, Angew. Chemie Int. Ed., 2005, 44, 7463-7465.

20 H. Mirabolghasemi, N. Liu, K. Lee and P. Schmuki, Chem. Commun., 2013, 49, 2067-9.

21 N. Liu, H. Mirabolghasemi, K. Lee, S. P. Albu, A. Tighineanu, M. Altomare and P. Schmuki, Faraday Discuss, 2013, 164, 107-116.

22 S. So, I. Hwang and P. Schmuki, Energy Environ. Sci., 2015, 8, 849-854.

23 S. So, K. Lee and P. Schmuki, J. Am. Chem. Soc., 2012, 134, 11316-11318.

24 I. Hwang, S. So, M. Mokhtar, A. Alshehri, S. a. Al-Thabaiti, A. Mazare and P. Schmuki, Chem. - A Eur. J., 2015, 21, 9204-9208. 
25 S. So, I. Hwang, F. Riboni, J. E. Yoo and P. Schmuki, Electrochem. commun., 2016, 71, 7378.

26 M. Fernández, J. Baonza, J. M. Albella and J. M. Martínez-Duart, Electrocompon. Sci. Technol., 1981, 7, 205-210.

27 F. Di Quarto, S. Piazza and C. Sunseri, J. Electroanal. Chem., 1988, 248, 99-115.

28 J.-F. Vanhumbeeck and J. Proost, Corros. Rev., 2009, 27, 117-204.

29 S. P. Albu, A. Ghicov, S. Aldabergenova, P. Drechsel, D. LeClere, G. E. Thompson, J. M. Macak and P. Schmuki, Adv. Mater., 2008, 20, 4135-4139.

30 S. Berger, S. P. Albu, F. Schmidt-Stein, H. Hildebrand, P. Schmuki, J. S. Hammond, D. F. Paul and S. Reichlmaier, Surf. Sci., 2011, 605, L57-L60.

31 W. Wei, S. Berger, C. Hauser, K. Meyer, M. Yang and P. Schmuki, Electrochem. commun., 2010, 12, 1184-1186.

32 M. Enachi, I. Tiginyanu, V. Sprincean and V. Ursaki, Phys. Status Solidi-Rapid Res. Lett., 2010, 4, 100-102.

33 T. Ruff, R. Hahn and P. Schmuki, Appl. Surf. Sci., 2011, 257, 8177-8181.

34 I. Barin, Thermochemical data of pure substances, VCH, Weinheim, Germany, 3rd edn., 1995.

35 U. Thanganathan and M. Nogami, Materials Challenges in Alternative and Renewable Energy, Wiley- VCH, 2010.

36 M. a Henderson, Surf. Sci., 1998, 400, 203-219.

37 U. Diebold, Surf. Sci. Rep., 2003, 48, 53-229.

38 R. Beranek and H. Kisch, Photochem. Photobiol. Sci., 2008, 7, 40-48.

39 R. Nakamura, A. Imanishi, K. Murakoshi and Y. Nakato, J. Am. Chem. Soc., 2003, 125, 7443-50.

40 P. a Connor, K. D. Dobson and a J. Mcquillan, Society, 1999, 2402-2408.

41 X. Jiang, Y. Wang, T. Herricks and Y. Xia, J. Mater. Chem., 2004, 14, 695.

42 M. Wei, H. Zhou, Y. Konishi, M. Ichihara, H. Sugiha and H. Arakawa, Inorg. Chem., 2006, 45, 5684-5690.

43 I. Dolamic and T. Bürgi, J. Catal., 2007, 248, 268-276.

44 Y.-K. Chen, Y.-F. Lin, Z.-W. Peng and J.-L. Lin, J. Phys. Chem. C, 2010, 114, 1772017727.

45 S. Vyazovkin, Anal. Chem., 2006, 78, 3875-3886.

46 S. Bauer, A. Pittrof, H. Tsuchiya and P. Schmuki, Electrochem. commun., 2011, 13, 538541. 


\section{Captions}

Figure 1. SEM top-view (first two columns) and cross-sectional (third column) images of (ac) double-walled NTs, (d-f) single-walled NTs obtained from DW tubes after mild annealing and chemical etching, and (g-i) tube-in-tube fabricated by anodizing at $10^{\circ} \mathrm{C}$. In particular, the first column shows amorphous samples, while the second and third columns report crystalline samples.

Figure 2. XRD patterns of (a) amorphous and (b) crystalline DW, SW and T-in-T layers. (c) Current-time behavior for anodization at $120 \mathrm{~V}$ in LA electrolyte at room temperature (blackcurve) leading to a double-walled morphology, and at $10^{\circ} \mathrm{C}$ (red curve) resulting in a tube-in-tube morphology after annelaing.

Figure 3. (a) $\mathrm{C}$ and $\mathrm{F}$ contents of the three investigated as-grown layers, determined by EDX measurements; (b-d) High resolution Auger Electron Spectroscopy (AES) horizontal scans of different elements ( $\mathrm{Ti}, \mathrm{C}$ and $\mathrm{F}$ ) recorded with as-formed $\mathrm{TiO}_{2}$ nanotube layers.

Figure 4. High Angle Annular Dark Field Scanning TEM (HAADF-STEM) images and C, F and $\mathrm{TiO}$ elemental mappings of (a) $\mathrm{DW}$, (b) $\mathrm{SW}$ and (c) $\mathrm{T}$-in-T(prec) $\mathrm{TiO}_{2} \mathrm{NT}$. All samples are amorphous.

Figure 5. F elemental TEM mappings of (a) as-formed and (b) crystalline T-in- $\mathrm{T} \mathrm{TiO}_{2} \mathrm{NTs}$. (c) HRTEM of a T-in-T nanotube formed after annealing.

Figure 6. Thermal desorption profiles for $\mathrm{m} / \mathrm{e}=18\left(\mathrm{H}_{2} \mathrm{O}\right)$ and $\mathrm{m} / \mathrm{e}=44\left(\mathrm{CO}_{2}\right)$ measured by Thermo-Gravimetric Analysis coupled with Mass Spectrometry (TGA-MS) for (a) double-walled, (b) single-walled, (c) T-in-T layers. (d) Comparison of the $\mathrm{CO}_{2}$ evolution peaks measured for the three different layers.

Figure 7. (a) FTIR spectra of as-grown SW, DW and T-in-T tubes. (b) FTIR spectra of conventional as-formed DW $\mathrm{TiO}_{2} \mathrm{NTs}$ (DW(ref)), of DW tubes annealed under mild conditions 
(DW(Ann150)), and of as-formed DW tubes without the washing-step in EtOH typically performed after anodization (DW(no EtOH)).

Figure 8. (a) Differential Scanning Calorimetry curves of SW, DW and T-in-T tubes. (b) DSC spectra recorded for conventional as-formed DW $\mathrm{TiO}_{2} \mathrm{NTs}(\mathrm{DW}(\mathrm{ref})$ ), of DW tubes annealed under mild conditions (DW(Ann150)), and of as-formed DW tubes without the washing-step in EtOH typically performed after anodization (DW(no EtOH)). 
Figure 1

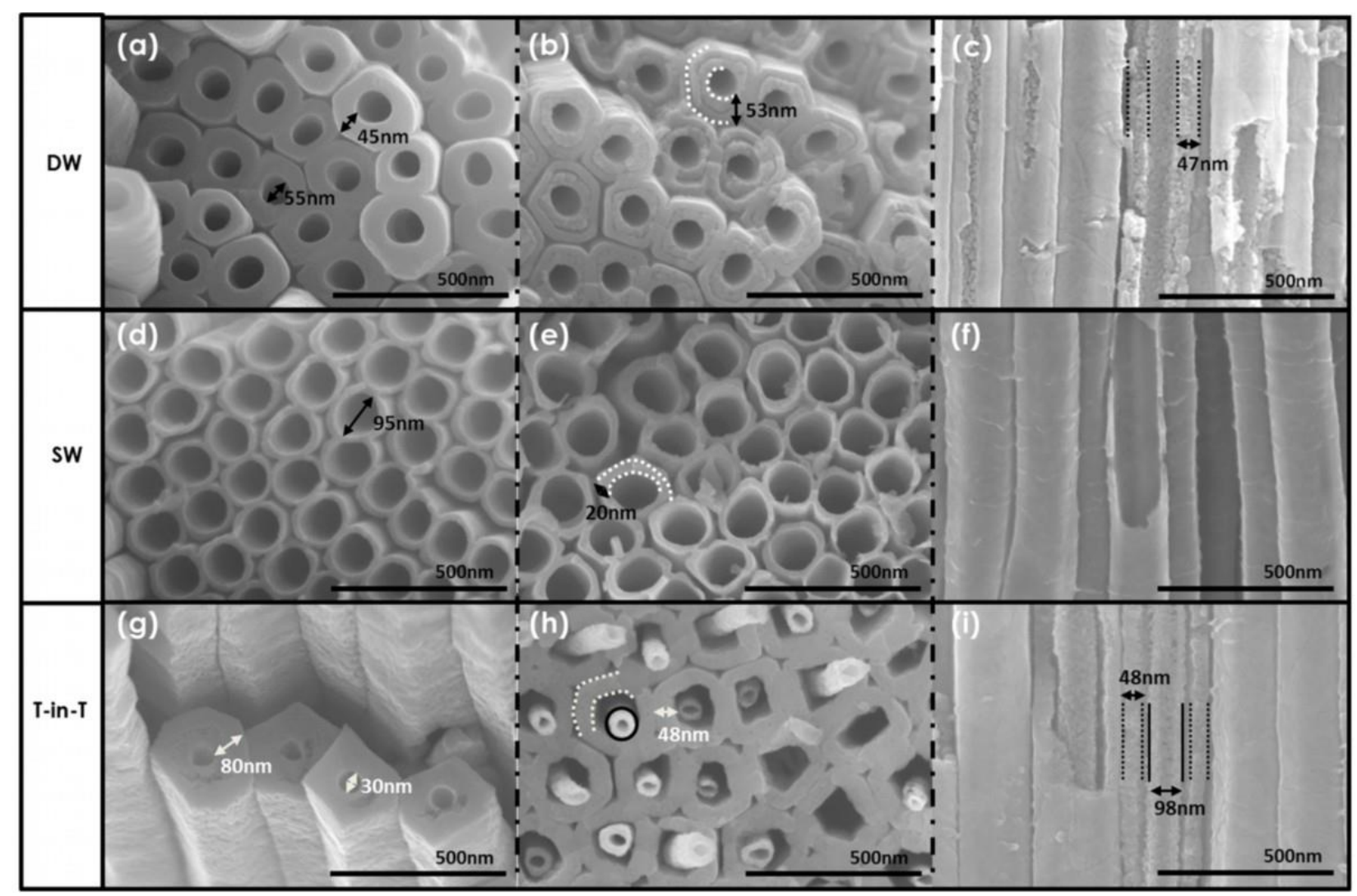


Figure 2
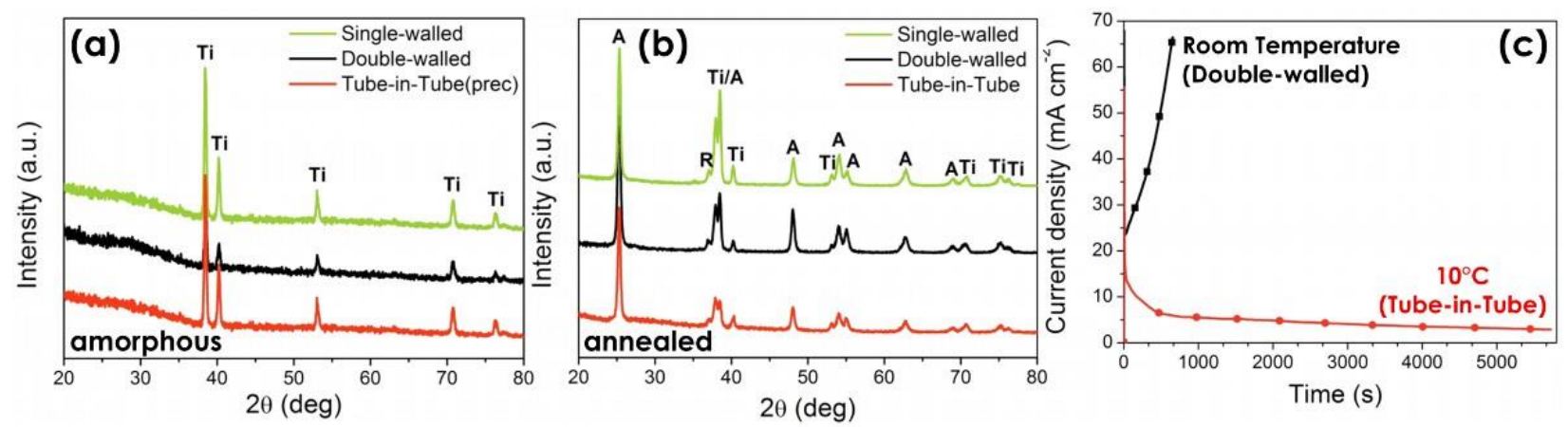
Figure 3

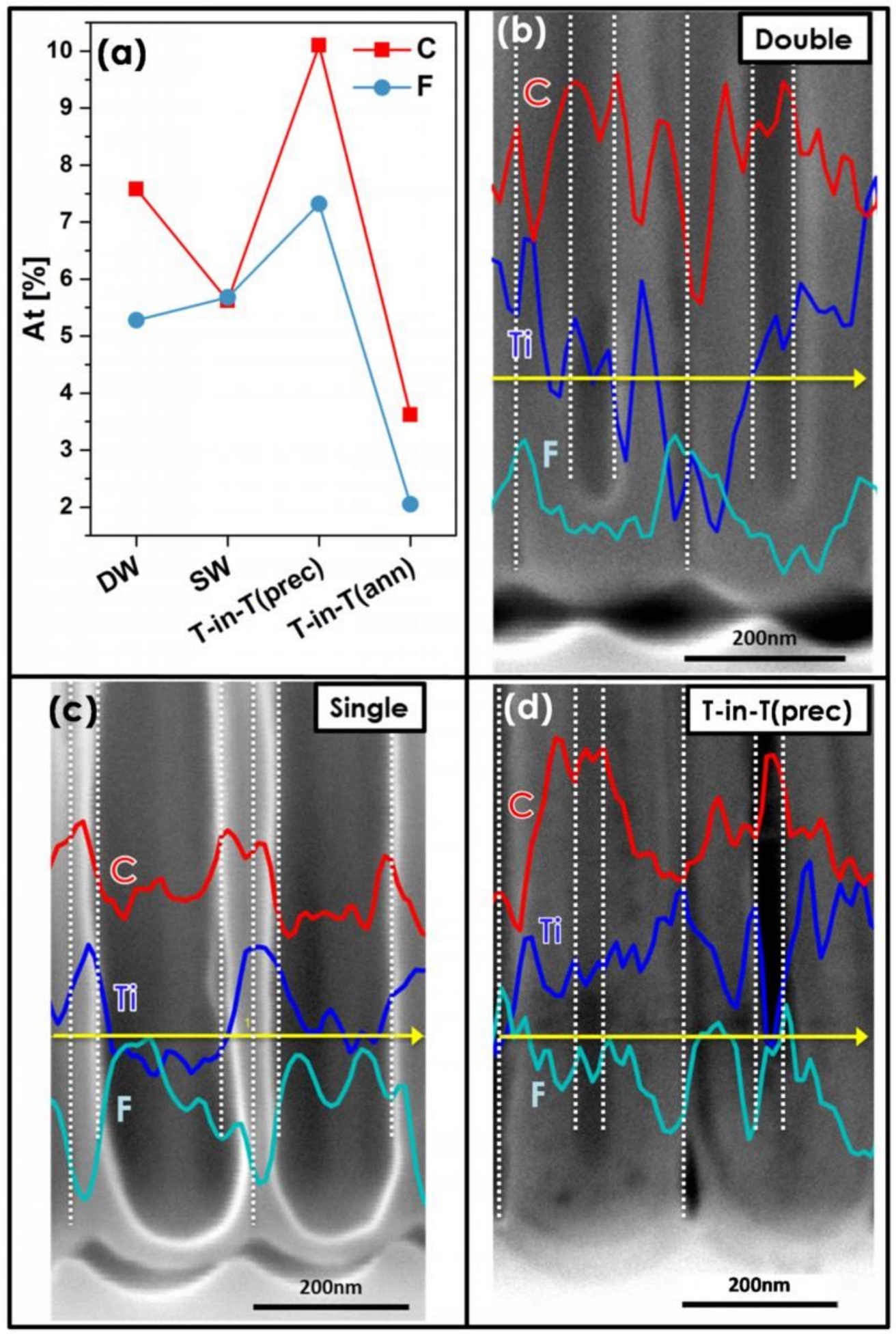


Figure 4

\section{(a) Double-walled}

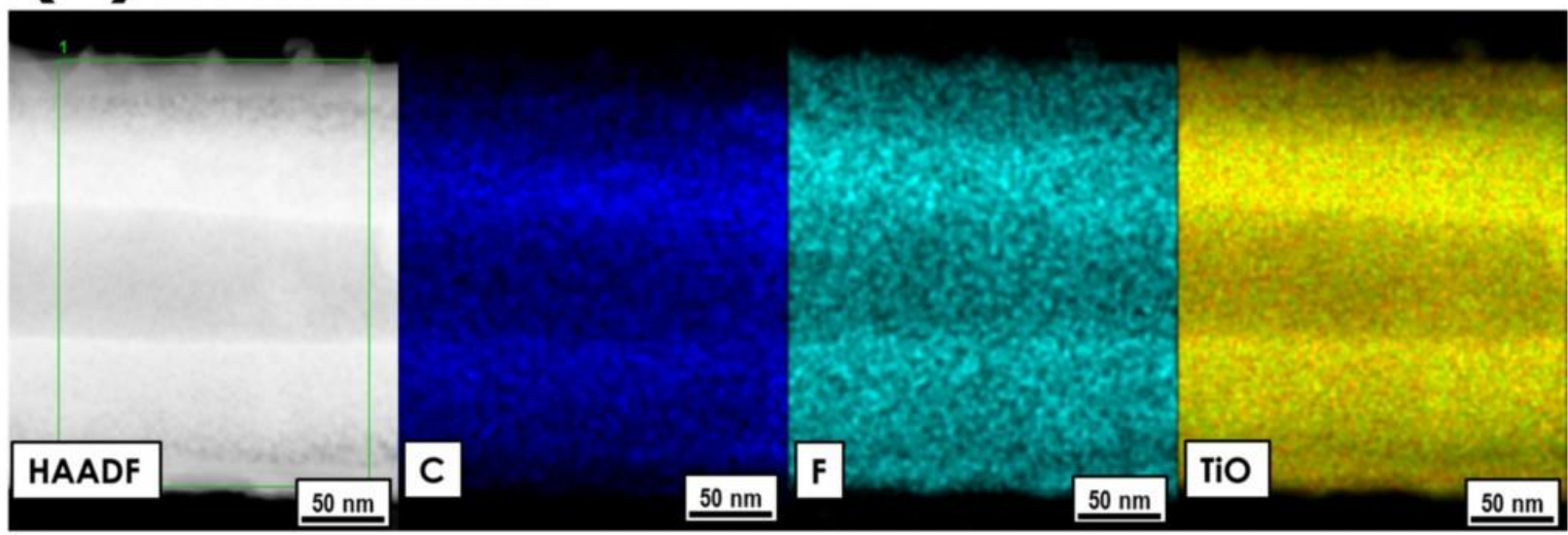

\section{(b) Single-walled}

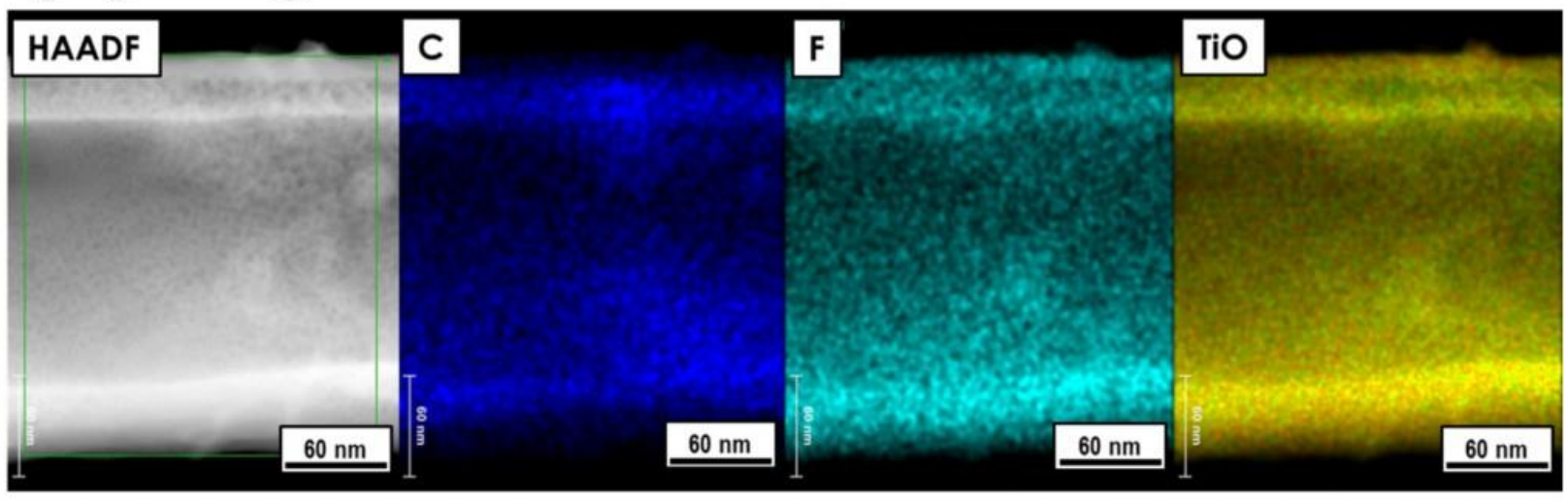

\section{(c) T-in-T(prec)}

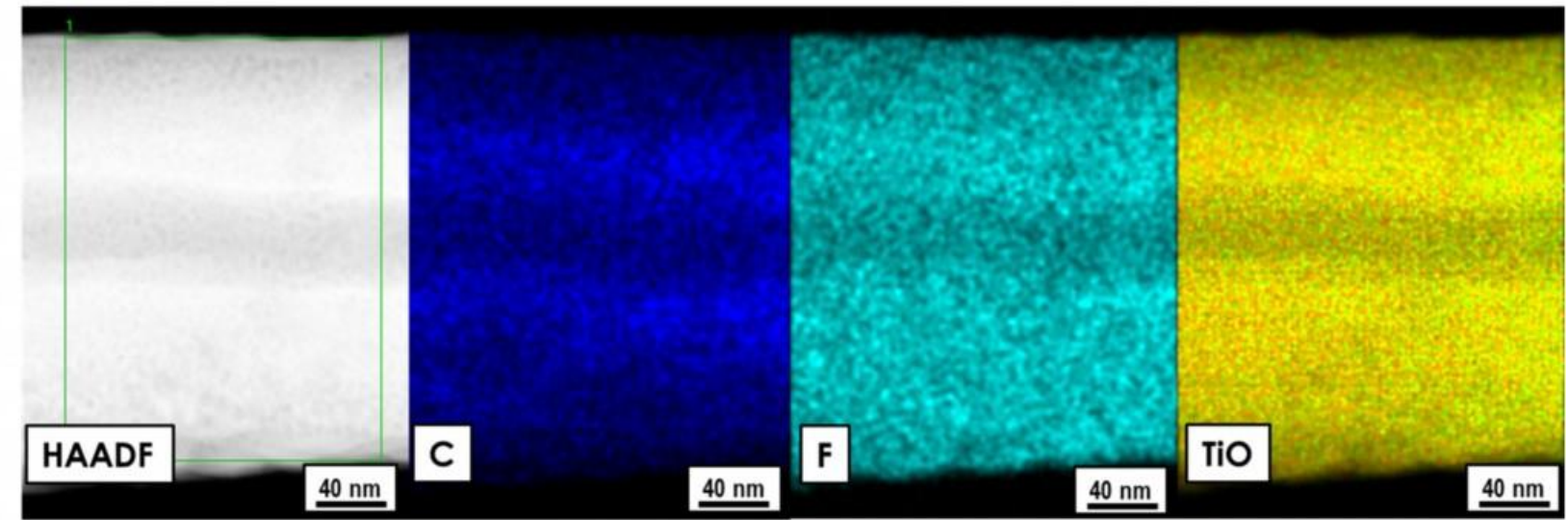


Figure 5
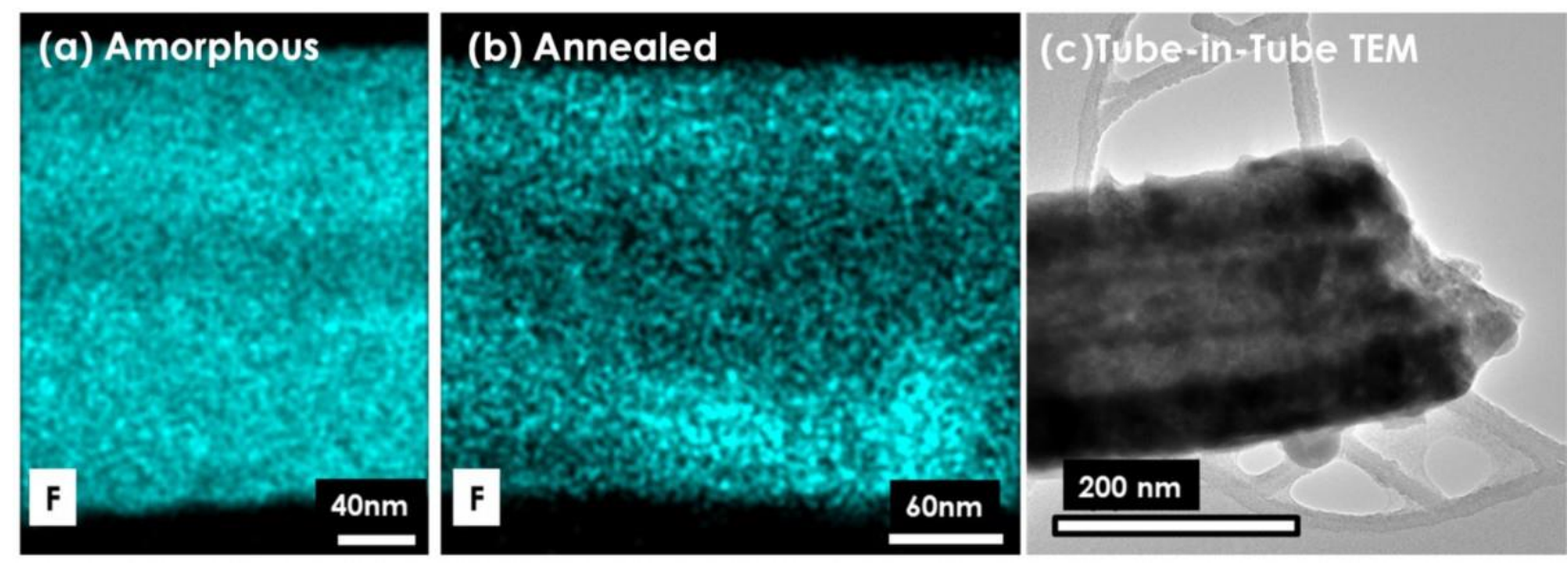


\section{Figure 6}
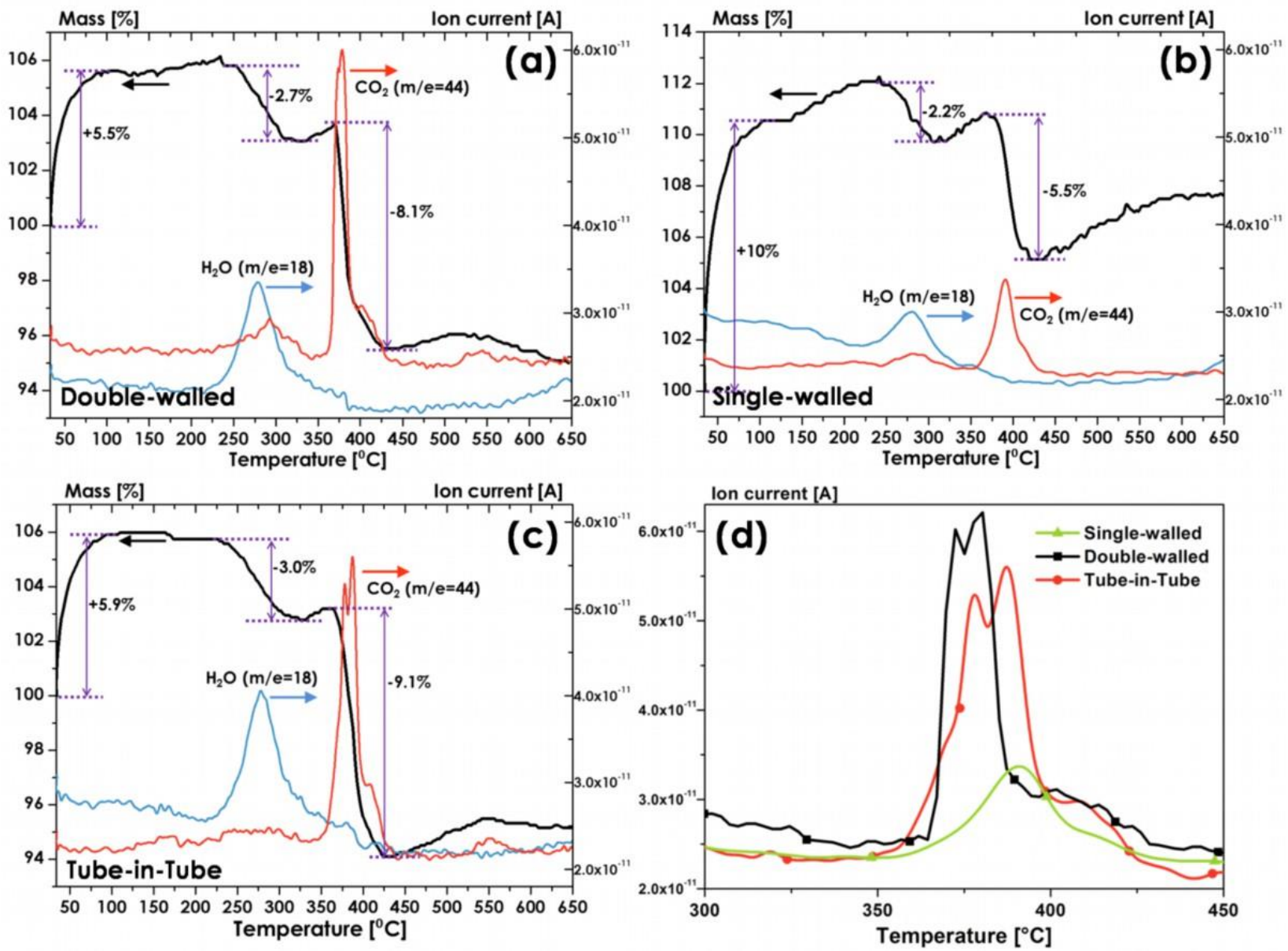
Figure 7

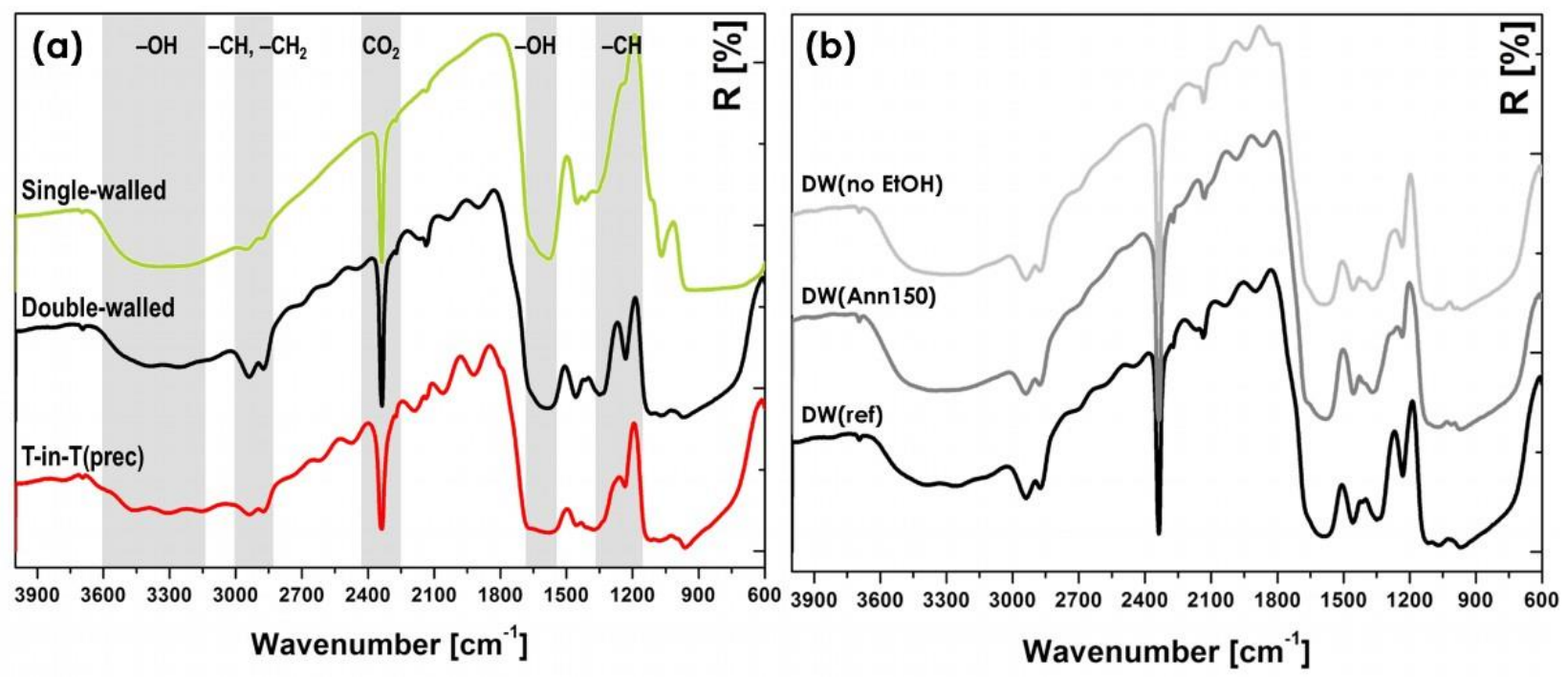


Figure 8
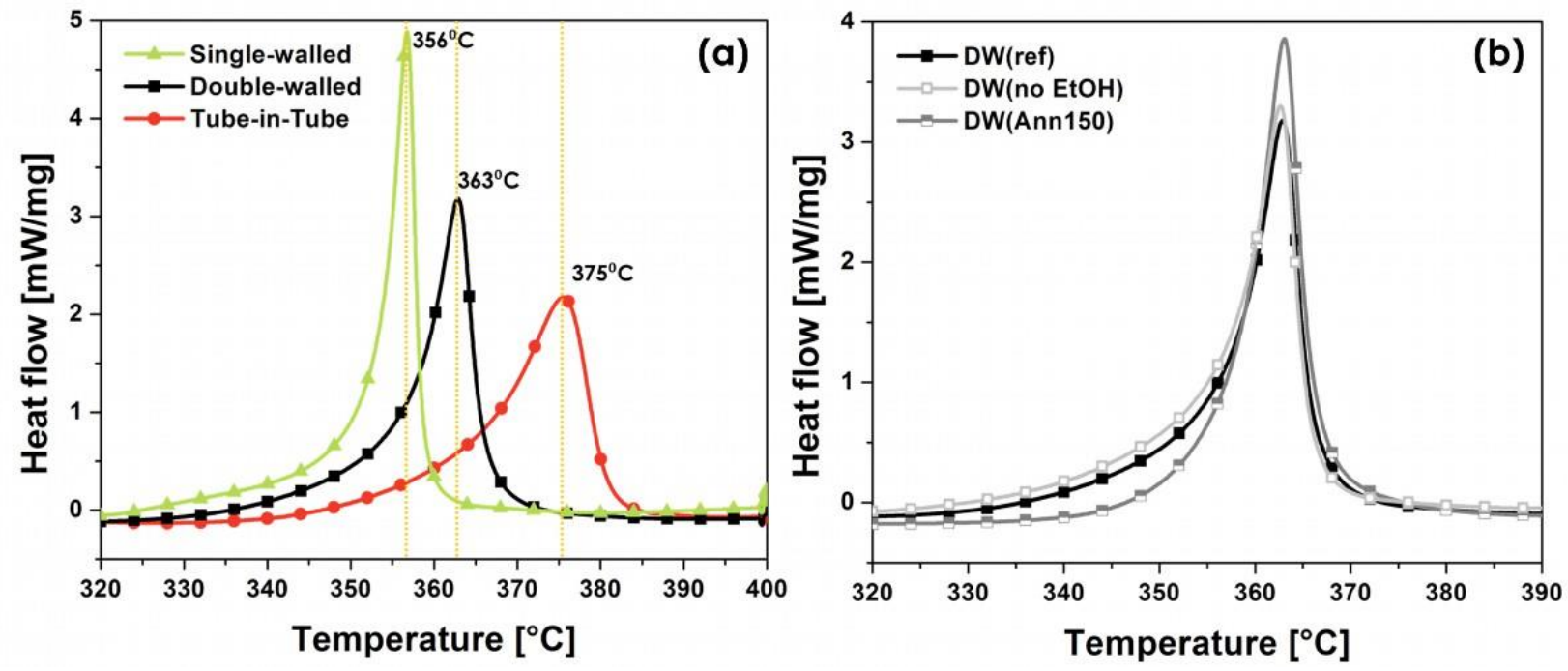